\title{
Stability and accuracy of active shielding for grounded capacitive sensors
}

\author{
Ferran Reverter ${ }^{1,2}$, Xiujun $\mathrm{Li}^{2}$ and Gerard C M Meijer ${ }^{2}$ \\ ${ }^{1}$ Castelldefels School of Technology (EPSC), Technical University of Catalonia (UPC), \\ Avda Canal Olimpic s/n, 08860 Castelldefels, Barcelona, Spain \\ ${ }^{2}$ Electronic Instrumentation Laboratory, Delft University of Technology (TU Delft), \\ Mekelweg 4, 2628 CD Delft, The Netherlands \\ E-mail: reverter@eel.upc.edu
}

Received 13 April 2006, in final form 26 July 2006

Published 28 September 2006

Online at stacks.iop.org/MST/17/2884

\begin{abstract}
Active shielding is commonly used to measure remote grounded capacitive sensors because it reduces the effects of both external noise/interference and parasitic capacitances of the shielded cable. However, due to active shielding, the measurement circuit can become unstable and inaccurate. This paper analyses these limitations theoretically and experimentally, and then provides guidelines for improving the performance of active shielding. One of the key points is the selection of the bandwidth of the amplifier that drives the shield of the coaxial cable. A wide bandwidth improves accuracy, but a narrow bandwidth improves stability. Therefore, there is a trade-off between stability and accuracy with respect to the bandwidth of the amplifier.
\end{abstract}

Keywords: active shielding, capacitive sensor, operational amplifier, stability, accuracy

(Some figures in this article are in colour only in the electronic version)

\section{Introduction}

Capacitive sensors are increasingly common in laboratory and industrial measurements because they can be built with affordable technologies, can be tailored to the geometry of different applications and have low power consumption [1]. This paper deals with interface circuits for grounded capacitive sensors, i.e. sensors in which one of the two electrodes is connected to the ground. Several humidity sensors, liquidlevel sensors [2, 3] and distance/proximity sensors [4] belong to this group.

In many industrial applications, the sensor is not close to its readout circuit. In these cases, to reduce the effects of external noise and interference, the sensor is connected to the circuit using a coaxial or shielded cable. There are two types of shielding: passive and active. In the case of passive shielding, the outer conductor is connected to the ground, as shown in figure 1(a). Regrettably, this shielding is not suitable for grounded capacitive sensors because the parasitic capacitance of the coaxial cable $\left(C_{\mathrm{p}}\right)$, whose value can be much greater than that of the sensor and depends on the environmental conditions, would be in parallel with the sensor $\left(C_{x}\right)[5]$.

In the case of active shielding, the outer conductor is driven at the same potential as that of the inner conductor by using a buffer amplifier [6, 7], as shown in figure $1(b)$. Here, on the one hand, external interferences are driven to ground through the low output impedance of the amplifier and, on the other hand, $C_{\mathrm{p}}$ ideally does not affect the measurement of $C_{x}$ because both cable conductors are at the same potential. Therefore, in principle, this technique solves the drawbacks of passive shielding. However, due to the parasitic components of the coaxial cable, the buffer amplifier has a positive feedback path that can bring about instability [8-11]. In addition, due to the limited bandwidth of the buffer amplifier, the inner and outer conductors of the coaxial cable are not at exactly the same potential. Consequently, the effect of $C_{\mathrm{p}}$ is not completely cancelled, thus limiting the accuracy of the measurement.

This paper analyses the stability and accuracy of the active shielding technique when it is applied in the measurement of grounded capacitive sensors. This analysis provides guidelines for selecting the bandwidth of the buffer amplifier. 


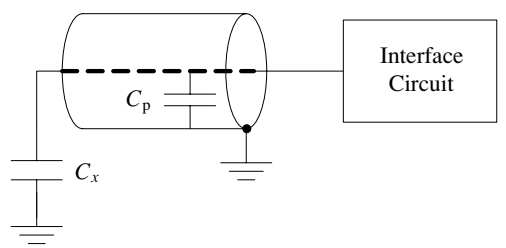

(a)

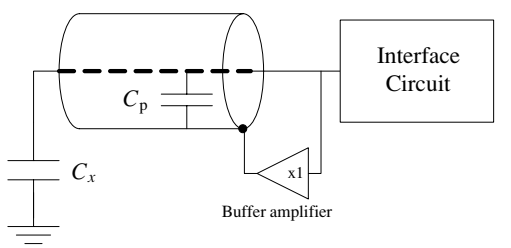

(b)

Figure 1. Measurement of a grounded capacitive sensor $\left(C_{x}\right)$ using $(a)$ passive shielding and $(b)$ active shielding.

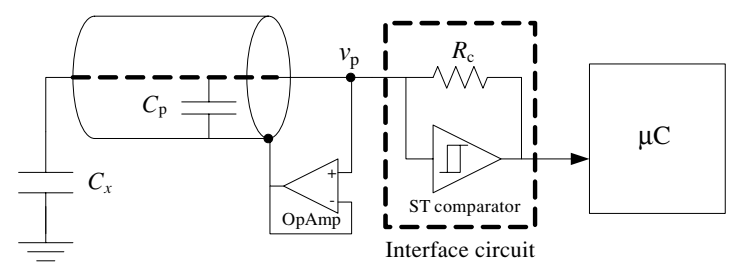

Figure 2. Measurement of a grounded capacitive sensor $\left(C_{x}\right)$ using active shielding and an RC oscillator as an interface circuit.

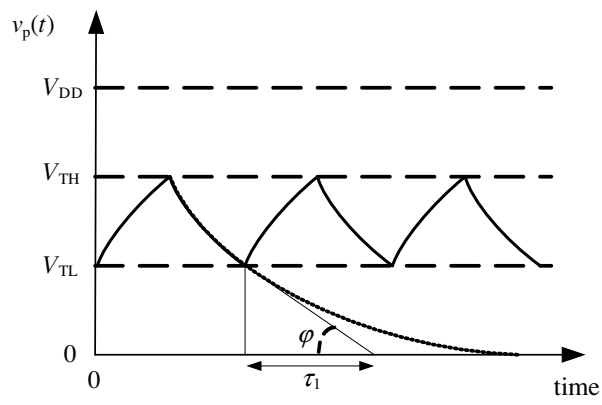

Figure 3. Waveform of the voltage $v_{\mathrm{p}}$ (figure 2) during the charging-discharging process.

\section{Circuit overview}

Grounded capacitive sensors are usually measured using $R C$ oscillator circuits, which can easily be implemented with, for instance, a 555 timer IC [1, 10] or a comparator-based relaxation oscillator $[4,10,11]$. Such an $R C$ oscillator basically relies on a Schmitt trigger (ST) comparator, which has an upper $\left(V_{\mathrm{TH}}\right)$ and a lower $\left(V_{\mathrm{TL}}\right)$ threshold voltage, a resistor $R_{\mathrm{c}}$ and the capacitive sensor $C_{x}$ itself, as shown in figure 2. The oscillator circuit works as follows. Let us consider that first the comparator has a high-level output voltage. Then, $C_{x}$ is charged towards the positive supply voltage $\left(V_{\mathrm{DD}}\right)$ through $R_{\mathrm{c}}$ and, hence, the voltage $v_{\mathrm{p}}$ increases exponentially with a time constant $\tau_{1}=R_{\mathrm{c}} C_{x}$. When $v_{\mathrm{p}}$ reaches $V_{\mathrm{TH}}$, the comparator is triggered. Afterwards, the comparator has a low-level output voltage and, hence, $C_{x}$ is discharged towards ground through $R_{\mathrm{c}}$ and $v_{\mathrm{p}}$ decreases exponentially. When $v_{\mathrm{p}}$ reaches $V_{\mathrm{TL}}$, the output of the comparator returns to its initial state and the process starts again. Figure 3 shows the resulting exponential waveform of $v_{\mathrm{p}}$ during the chargingdischarging process.

Under ideal conditions, the output of the comparator is a square-wave signal whose period is proportional to $C_{x}$ [12]. This time-based output signal can be measured directly using a digital system, such as a microcontroller, as shown in figure 2.
The circuit can also include one or more reference capacitors (which are selected by an analogue multiplexer) to compensate for the deviation of the resistor, the supply voltage and the threshold voltages [11].

The function of the buffer amplifier that drives the shield of the coaxial cable can easily be carried out by an operational amplifier (OpAmp) configured as a voltage follower, as shown in figure 2. In order to drive the shield correctly, the OpAmp must (a) be unity-gain stable, (b) have a slew rate greater than the maximal slope of the exponential signal (figure 3) and (c) have a rail-to-rail input/output topology or, at least, the common-mode input voltage range and the output swing must include the voltage range of the exponential signal (figure 3). Regrettably, it is not clear how to select the bandwidth of the OpAmp in order to achieve a good performance. Next, we will provide rules for bandwidth selection.

\section{Theoretical analysis}

The performance of active shielding is analysed using the circuit shown in figure 4(a). This circuit uses an input voltage $v_{\text {in }}$ instead of the voltage provided by the ST comparator in figure 2 . The voltage to be analysed is $v_{\mathrm{p}}$, which is the voltage that will then be compared to the threshold voltages of the ST comparator.

Figure $4(b)$ shows the equivalent circuit of figure $4(a)$ when the parasitic components of the interconnection cable are taken into account. The capacitor $C_{\mathrm{p}}$ represents the capacitance between the inner conductor and the shield of the coaxial cable, $L_{\mathrm{p}}$ is the inductance of the current loop between the circuit and $C_{x}$, and $R_{\mathrm{p}}$ is the resistance of the interconnection conductors. Let us assume that $R_{\mathrm{p}}, C_{\mathrm{p}}$ and $L_{\mathrm{p}}$ depend linearly on the length $\ell$ of the interconnection cable and define $r_{\mathrm{p}}, c_{\mathrm{p}}$ and $l_{\mathrm{p}}$ as the parasitic resistance, capacitance and inductance per unit length, respectively.

With regard to the OpAmp, we apply the macro-model shown in figure 5, which includes two voltage-controlled voltage sources (VCVS). The first VCVS has a gain of $A_{0}$, which is the differential dc gain of the OpAmp, whereas the second VCVS has a unity gain. The resistor $R_{\text {opa }}$ and the capacitor $C_{\text {opa }}$ model the frequency limitations. As a result, the OpAmp has a dominant pole $\omega_{\mathrm{a}}=2 \pi f_{\mathrm{a}}=\left(R_{\text {opa }} C_{\text {opa }}\right)^{-1}$ and a unity-gain bandwidth $\omega_{\mathrm{b}}=2 \pi f_{\mathrm{b}}=A_{0} \omega_{\mathrm{a}}$. The model in figure 5 also takes into account the output resistance $R_{\mathrm{o}}$.

A voltage follower based on the OpAmp shown in figure 5 behaves as a unity-gain first-order system with a time constant $\tau_{\mathrm{b}}=1 / \omega_{\mathrm{b}}$. If the threshold voltages of the ST comparator are close to each other, then the input signal of the voltage follower shows an almost triangular wave shape, as shown in figure 3. The response of a unity-gain first-order system to a ramp input 


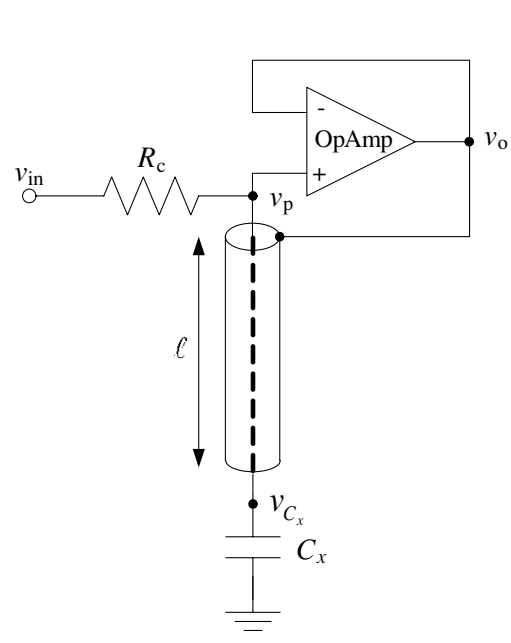

(a)

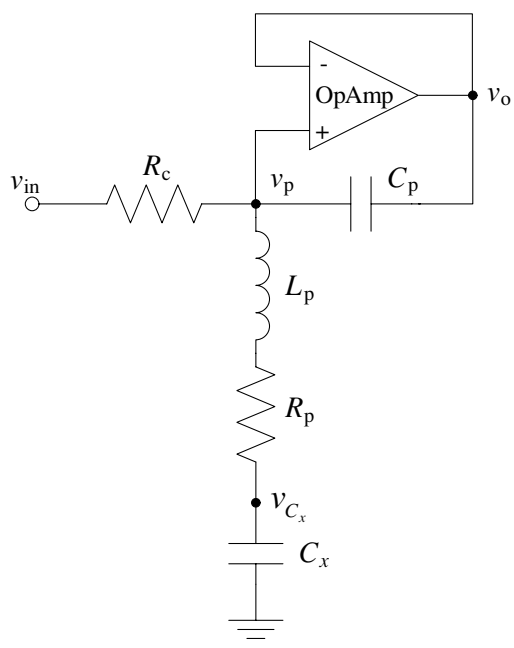

(b)

Figure 4. (a) Simplified circuit used to analyse the performance of active shielding. (b) Equivalent circuit that includes the parasitic components of the interconnection cable.

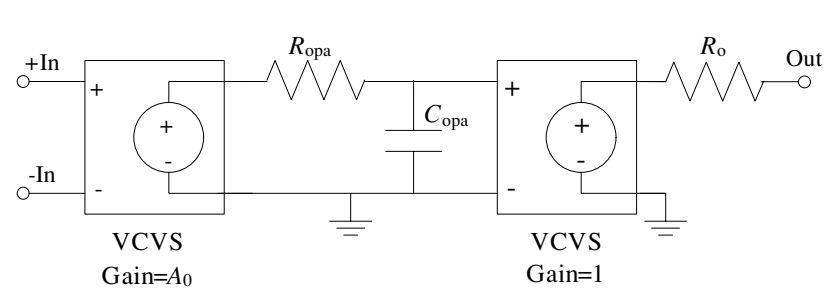

Figure 5. Macro-model of the OpAmp that considers the dominant-pole open-loop response and the output resistance $R_{\mathrm{o}}$.

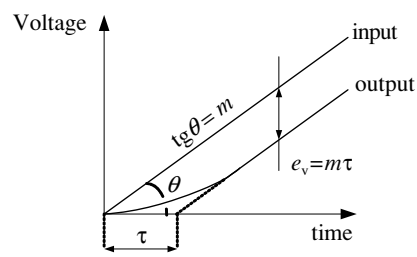

(a)

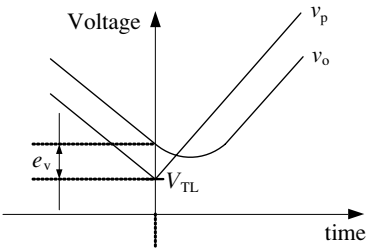

(b)
Figure 6. (a) Response of a unity-gain first-order system to a ramp input signal. (b) Waveforms of the input $\left(v_{\mathrm{p}}\right)$ and output $\left(v_{\mathrm{o}}\right)$ signals of the voltage follower at the beginning of the charging stage.

signal is shown in figure 6(a) [10], where $m$ is the slew rate of the input signal, $\tau$ is the time constant of the system and $e_{\mathrm{v}}$, which is equal to $m \tau$, is the voltage difference between the input and the output. Therefore, the output signal of the voltage follower will differ slightly from the input signal. As an example, figure $6(b)$ shows the input $\left(v_{\mathrm{p}}\right)$ and output $\left(v_{\mathrm{o}}\right)$ signals of the voltage follower at the beginning of the charging stage.

Taking into account the above models, next we analyse theoretically the stability and accuracy of the circuit.

\subsection{Stability}

A systematic analysis of the circuit in figure $4(b)$ provides the following fourth-order transfer function between $V_{\mathrm{p}}(s)$ and
$V_{\text {in }}(s)$ :

$$
H(s)=\frac{V_{\mathrm{p}}(s)}{V_{\mathrm{in}}(s)}=\frac{Q(s)\left[\left(R_{\mathrm{o}} C_{\mathrm{p}} s+1\right)\left(s+\omega_{\mathrm{a}}\right)+\omega_{\mathrm{b}}\right]}{P(s)\left(s+\omega_{\mathrm{a}}\right)+\left(Q(s)+R_{\mathrm{c}} C_{x} s\right) \omega_{\mathrm{b}}},
$$

where

$$
Q(s)=C_{x} L_{\mathrm{p}} s^{2}+R_{\mathrm{p}} C_{x} s+1
$$

$$
\begin{aligned}
& P(s)=C_{\mathrm{p}} C_{x} L_{\mathrm{p}}\left(R_{\mathrm{o}}+R_{\mathrm{c}}\right) s^{3} \\
& \quad+C_{x}\left[L_{\mathrm{p}}+C_{\mathrm{p}}\left(R_{\mathrm{o}} R_{\mathrm{p}}+R_{\mathrm{c}} R_{\mathrm{p}}+R_{\mathrm{c}} R_{\mathrm{o}}\right)\right] s^{2} \\
& \quad+\left(R_{\mathrm{o}} C_{\mathrm{p}}+R_{\mathrm{p}} C_{x}+R_{\mathrm{c}} C_{x}+R_{\mathrm{c}} C_{\mathrm{p}}\right) s+1 .
\end{aligned}
$$

If we consider some practical relations between the parameters (e.g. $R_{\mathrm{c}} \gg R_{\mathrm{o}}, R_{\mathrm{c}} \gg R_{\mathrm{p}}, \omega_{\mathrm{b}} \gg \omega_{\mathrm{a}}$ ), then the denominator polynomial $d(s)$ of equation (1) is simplified to

$$
\begin{aligned}
d(s) & \approx R_{\mathrm{c}} C_{\mathrm{p}} C_{x} L_{\mathrm{p}} s^{4}+R_{\mathrm{c}} C_{\mathrm{p}} C_{x}\left(R_{\mathrm{p}}+R_{\mathrm{o}}\right) s^{3} \\
& +\left[R_{\mathrm{c}}\left(C_{\mathrm{p}}+C_{x}\right)+C_{x} L_{\mathrm{p}} \omega_{\mathrm{b}}\right] s^{2}+\left(1+R_{\mathrm{c}} C_{x} \omega_{\mathrm{b}}\right) s+\omega_{\mathrm{b}} .
\end{aligned}
$$

By applying the Routh-Hurwitz stability criterion [13] in equation (4), we obtain the following stability condition:

$$
f_{\mathrm{b}}<f_{\text {stab }}=\frac{1}{2 \pi} \frac{R_{\mathrm{c}}\left(C_{\mathrm{p}}+C_{x}\right)\left(R_{\mathrm{p}}+R_{\mathrm{o}}\right)-L_{\mathrm{p}}}{C_{x} L_{\mathrm{p}}\left(R_{\mathrm{c}}-R_{\mathrm{p}}-R_{\mathrm{o}}\right)},
$$

where the frequency $f_{\mathrm{stab}}$, which is determined by the components of the circuit in figure $4(b)$, is defined as the maximal allowable bandwidth of the OpAmp to guarantee stability.

Figure 7 depicts the value of $f_{\text {stab }}$ as calculated from equation (5) versus $C_{x}$ for different lengths of the coaxial cable. We consider $r_{\mathrm{p}}=1.0 \Omega \mathrm{m}^{-1}, c_{\mathrm{p}}=100 \mathrm{pF} \mathrm{m}^{-1}$ and $l_{\mathrm{p}}=$ $1.0 \mu \mathrm{H} \mathrm{m}^{-1}$, which are the features of the interconnection cable used in the experimental setup, and $R_{\mathrm{c}}=100 \mathrm{k} \Omega$. The output resistance of an OpAmp generally ranges from $50 \Omega$ to $200 \Omega$ [14]. Here we apply the minimal value (i.e. $R_{\mathrm{o}}=50 \Omega$ ) since, according to equation (5), this is the worst case in terms of stability. Applying the minimal value of $R_{\mathrm{o}}$ to estimate $f_{\text {stab }}$ can bring us to reject an OpAmp that could be used, but this is much better than to accept an OpAmp that makes the circuit unstable. Figure 7 shows that the greater either $C_{x}$ or $\ell$, the 


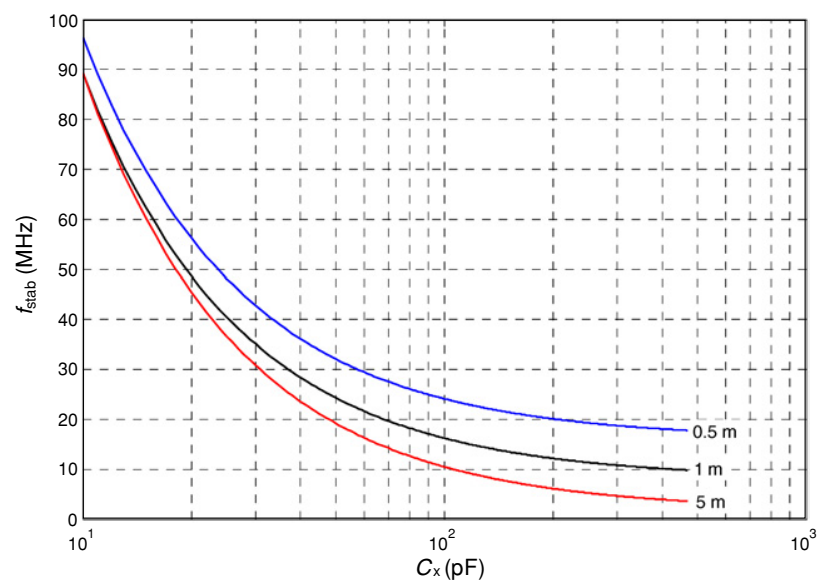

Figure 7. Maximal allowable bandwidth $\left(f_{\text {stab }}\right)$ of the OpAmp to guarantee stability versus $C_{x}$ for different lengths of the coaxial cable.

smaller $f_{\text {stab }}$ and, hence, the smaller the maximal allowable bandwidth of the OpAmp. For instance, for $C_{x}=100 \mathrm{pF}$ and $\ell=1 \mathrm{~m}$, the circuit is stable when $f_{\mathrm{b}}<16 \mathrm{MHz}$.

Equation (5) shows that practically $f_{\text {stab }}$ is inversely proportional to the parasitic inductance $L_{\mathrm{p}}$. Therefore, we can improve the stability of the circuit by decreasing $L_{\mathrm{p}}$, i.e. by decreasing the area of the current loop between the circuit and $C_{x}$ [15]. On the other hand, as either $C_{\mathrm{p}}$ or the factor $R_{\mathrm{p}}+$ $R_{\mathrm{o}}$ increases, so does $f_{\mathrm{stab}}$. Accordingly, a capacitor placed in parallel with $C_{\mathrm{p}}$ and/or a resistor added in series with either $R_{\mathrm{p}}$ or $R_{\mathrm{o}}$ should improve stability.

\subsection{Accuracy}

Let us first evaluate the circuit in figure 4(a) excluding the buffer amplifier and the coaxial cable, i.e. a simple $R C$ circuit with a time constant $\tau_{1}=R_{\mathrm{C}} C_{x}$. Under these conditions, the voltages $v_{\mathrm{p}}$ and $v_{C_{x}}$ correspond to the same point. A systematic analysis of this circuit by assuming $v_{C_{x}}(0)=V_{\mathrm{TL}}$ (figure 3 ) provides, in the Laplace domain,

$$
V_{\mathrm{p}}(s)=\frac{1}{s+\omega_{1}}\left[\omega_{1} V_{\mathrm{in}}(s)+V_{\mathrm{TL}}\right],
$$

where $\omega_{1}=1 / \tau_{1}$. During the charging stage, the input voltage shows a step of magnitude $V_{\mathrm{DD}}$, i.e. $V_{\mathrm{in}}(s)=V_{\mathrm{DD}} / s$. Then, transforming equation (6) into the time domain yields the following transient response:

$$
v_{\mathrm{p}}(t)=V_{\mathrm{DD}}+\left(V_{\mathrm{TL}}-V_{\mathrm{DD}}\right) \exp \left(-\omega_{1} t\right) .
$$

From equation (7), the time interval $t_{\mathrm{ch}}$ needed to charge $C_{x}$ through $R_{\mathrm{c}}$ from $V_{\mathrm{TL}}$ to $V_{\mathrm{TH}}$ equals

$$
t_{\mathrm{ch}}=\tau_{1} \ln \left[\frac{V_{\mathrm{DD}}-V_{\mathrm{TL}}}{V_{\mathrm{DD}}-V_{\mathrm{TH}}}\right] \text {. }
$$

Let us now analyse the effect of active shielding on the time interval $t_{\mathrm{ch}}$. Firstly, if the stability of the circuit is guaranteed, the inaccuracy can be evaluated by applying a simplified circuit model. In relation to the interconnection cable (figure 4(b)), the effects of $L_{\mathrm{p}}$ and $R_{\mathrm{p}}$ are neglected; the capacitor $C_{\mathrm{p}}$ has a dominant effect on the accuracy, which will be proved experimentally in section 4 . With regard to the OpAmp (figure 5), the effect of $R_{\mathrm{o}}$ is also neglected. Secondly,

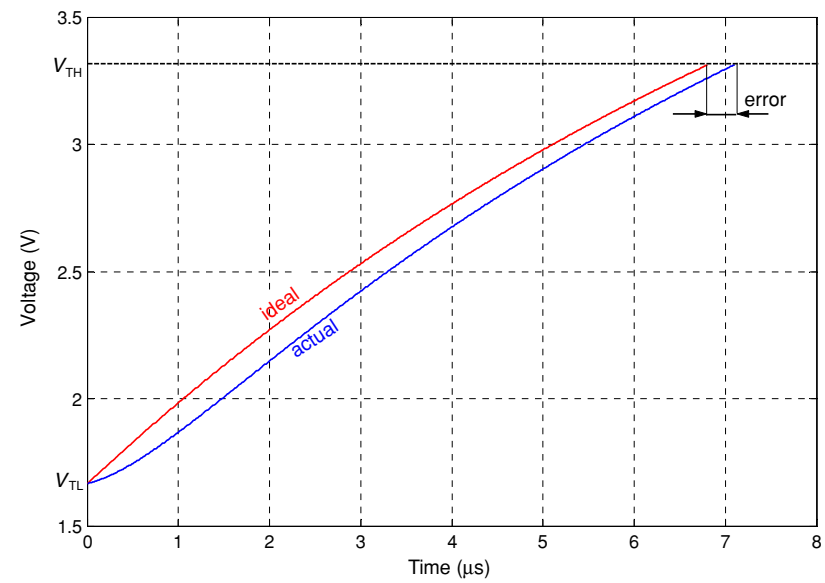

Figure 8. Ideal and actual exponential waveform of $v_{\mathrm{p}}$ during the charging stage.

the initial conditions here are $v_{C_{x}}(0)=V_{\mathrm{TL}}, v_{C_{\text {opa }}}(0)=V_{\mathrm{TL}}+e_{\mathrm{v}}$ and $v_{C_{\mathrm{p}}}(0)=-e_{\mathrm{v}}$, where $e_{\mathrm{v}}$ is the voltage difference between the input and the output of the OpAmp due to its limited bandwidth. Just when the discharging signal reaches $V_{\mathrm{TL}}$, the slew rate is $m=\tan \varphi=V_{\mathrm{TL}} / \tau_{1}$ (figure 3 ) and, hence, the voltage error is $e_{\mathrm{v}}=\left(\omega_{1} / \omega_{\mathrm{b}}\right) V_{\mathrm{TL}}$ (figure $\left.6(b)\right)$. Taking into account the above considerations, the initial conditions and $\omega_{\mathrm{b}} \gg \omega_{\mathrm{a}}$, we find, in the Laplace domain,

$$
\begin{aligned}
& V_{\mathrm{p}}(s) \approx \frac{1}{G(s)}\left[\left(s+\omega_{\mathrm{b}}\right) V_{\mathrm{in}}(s)+s R_{\mathrm{c}}\left(C_{\mathrm{p}}+C_{x}\right) V_{\mathrm{TL}}\right. \\
& \left.\quad+\omega_{\mathrm{b}} R_{\mathrm{c}}\left(V_{\mathrm{TL}} C_{x}-e_{\mathrm{v}} C_{\mathrm{p}}\right)\right],
\end{aligned}
$$

where

$$
G(s)=s^{2} R_{\mathrm{c}}\left(C_{\mathrm{p}}+C_{x}\right)+s\left(1+\omega_{\mathrm{b}} R_{\mathrm{c}} C_{x}\right)+\omega_{\mathrm{b}} .
$$

Then, for $V_{\text {in }}(s)=V_{\mathrm{DD}} / s$, transforming equation (9) into the time domain yields

$$
\begin{aligned}
v_{\mathrm{p}}(t) & =\left(\frac{k_{1}}{s_{1}-s_{2}}+\frac{k_{2}+V_{\mathrm{TL}} s_{1}^{2}}{\left(s_{1}-s_{2}\right) s_{1}}\right) \exp \left(s_{1} t\right) \\
& -\left(\frac{k_{1}}{s_{1}-s_{2}}+\frac{k_{2}+V_{\mathrm{TL}} s_{2}^{2}}{\left(s_{1}-s_{2}\right) s_{2}}\right) \exp \left(s_{2} t\right)+\frac{k_{2}}{s_{1} s_{2}},
\end{aligned}
$$

where $s_{1}$ and $s_{2}$ are the roots of the equation $G(s)=0$, and

$$
\begin{gathered}
k_{1}=\frac{V_{\mathrm{DD}}+\omega_{\mathrm{b}} R_{\mathrm{c}}\left(V_{\mathrm{TL}} C_{x}-e_{\mathrm{v}} C_{\mathrm{p}}\right)}{R_{\mathrm{c}}\left(C_{\mathrm{p}}+C_{x}\right)}, \\
k_{2}=\frac{V_{\mathrm{DD}} \omega_{\mathrm{b}}}{R_{\mathrm{c}}\left(C_{\mathrm{p}}+C_{x}\right)} .
\end{gathered}
$$

The ideal and actual exponential waveform of $v_{\mathrm{p}}$ (described by equations (7) and (11) respectively) are represented in figure 8 for $C_{x}=100 \mathrm{pF}, R_{\mathrm{c}}=100 \mathrm{k} \Omega$, $V_{\mathrm{DD}}=5 \mathrm{~V}, V_{\mathrm{TL}}=V_{\mathrm{DD}} / 3, V_{\mathrm{TH}}=2 V_{\mathrm{DD}} / 3, f_{\mathrm{b}}=500 \mathrm{kHz}$ and $C_{\mathrm{p}}=100 \mathrm{pF}$. Except for the value of $f_{\mathrm{b}}$, which is small so its effects can be seen more clearly, the rest of the values are usual. Figure 8 shows that, due to the limited bandwidth of the OpAmp and the effect of $C_{\mathrm{p}}$, the transient response is slower and the charging time is longer. As a result, there is an error in the charging time.

Equation (11) does not have a symbolical solution for $t_{\mathrm{ch}}$. Consequently, the actual value of $t_{\mathrm{ch}}$ and its relative error (using the value provided by (8) as a reference value) have 


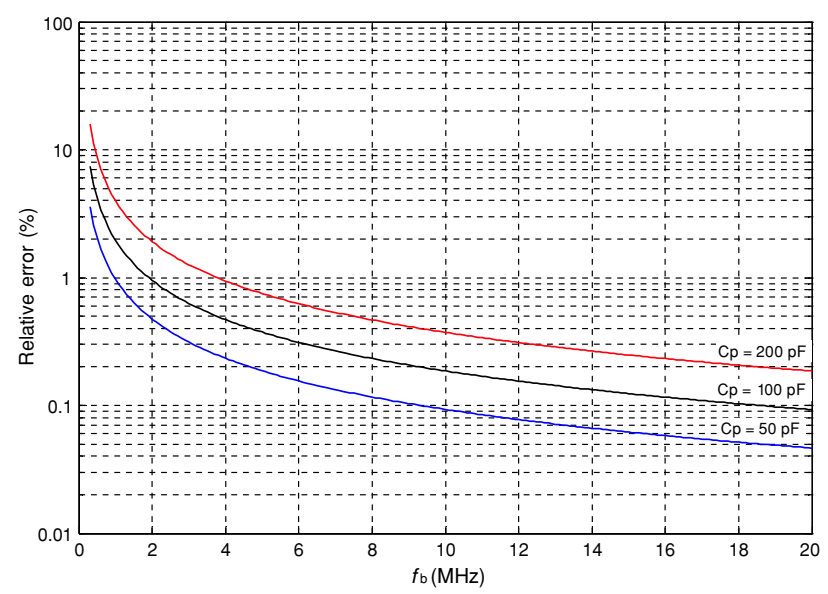

Figure 9. Relative error in the charging time as caused by the limited bandwidth of the OpAmp and the parasitic capacitance of the coaxial cable.

been calculated numerically. Figure 9 shows the relative error of $t_{\mathrm{ch}}$ versus $f_{\mathrm{b}}$ for different values of $C_{\mathrm{p}}(50 \mathrm{pF}, 100 \mathrm{pF}$ and $200 \mathrm{pF}$, which correspond to $0.5 \mathrm{~m}, 1 \mathrm{~m}$ and $2 \mathrm{~m}$ of cable length respectively) when $C_{x}=100 \mathrm{pF}, R_{\mathrm{c}}=100 \mathrm{k} \Omega, V_{\mathrm{DD}}=$ $5 \mathrm{~V}, V_{\mathrm{TL}}=V_{\mathrm{DD}} / 3$ and $V_{\mathrm{TH}}=2 V_{\mathrm{DD}} / 3$. From figure 9, we can conclude that, whenever the circuit is stable, the greater the value of $f_{\mathrm{b}}$ or the shorter the length of the coaxial cable, the smaller the relative error in the charging time.

If the oscillator has symmetrical threshold voltages with respect to $V_{\mathrm{DD}} / 2$, the charging and discharging times are the same length. In addition, the time errors as caused by active shielding are equal for both time intervals. Consequently, the results shown in figure 9 can also be applied to estimate the overall relative error for the whole period of the oscillator output signal. For instance, for $\ell=1 \mathrm{~m}$ and $f_{\mathrm{b}}=10 \mathrm{MHz}$, the period of the output signal has a relative error of $0.2 \%$.

An error in the period of the oscillator output signal directly brings about an error in the estimation of $C_{x}$ and, hence, in the estimation of the measurand. Auto-calibration methods, such as the three-signal technique [16], cannot compensate for this error because the reference components, which, in a practical setup, are built together with the interface circuit, do not suffer from the influence of active shielding. Therefore, it is advisable to reduce this error by selecting an OpAmp with a large $f_{\mathrm{b}}$ value. However, at the same time, the circuit must be stable and, hence, $f_{\mathrm{b}}<f_{\text {stab }}$ (figure 7). Consequently, there is an optimal range of $f_{\mathrm{b}}$ values that provide both stability and accuracy.

\section{Experimental results}

The performance of active shielding was experimentally tested in terms of stability and accuracy. To evaluate the effect of the bandwidth of the OpAmp, we selected six commercial OpAmps with different bandwidths. All of these OpAmps can operate at a single supply voltage and fulfil the requirements listed in section 2. Table 1 lists the OpAmps and their nominal and actual $f_{\mathrm{b}}$ values. The actual value was measured using a sinusoidal frequency sweep. Except for OPA743, the actual value of $f_{\mathrm{b}}$ was always higher than the nominal one.

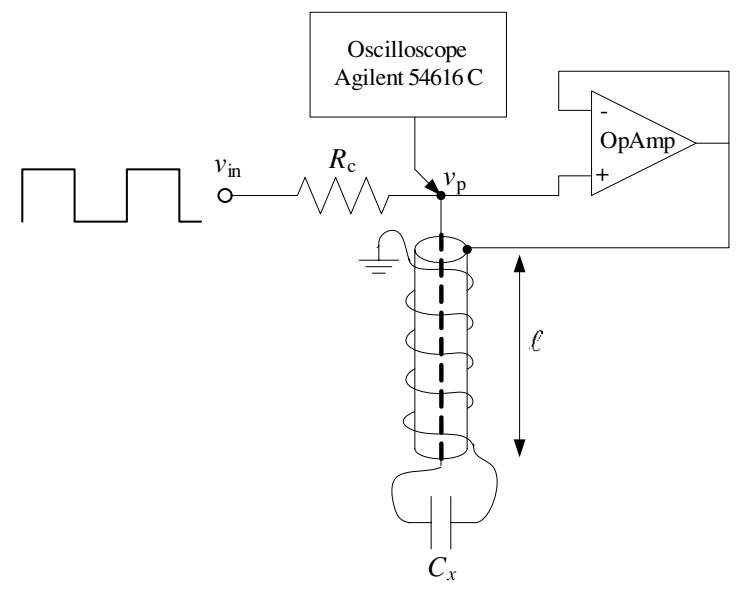

Figure 10. Experimental setup used to test the stability of active shielding.

Table 1. Nominal and measured unity-gain bandwidths $\left(f_{\mathrm{b}}\right)$ of the tested OpAmps.

\begin{tabular}{lcc}
\hline OpAmp & $\begin{array}{l}\text { Nominal } \\
f_{\mathrm{b}}(\mathrm{MHz})\end{array}$ & $\begin{array}{l}\text { Actual } \\
f_{\mathrm{b}}(\mathrm{MHz})\end{array}$ \\
\hline OPA344 & 1 & 1.2 \\
OPA337 & 3 & 3.6 \\
OPA743 & 7 & 5.4 \\
TLC071 & 10 & 11 \\
AD8655 & 28 & 30 \\
OPA350 & 38 & 64 \\
\hline
\end{tabular}

\subsection{Stability}

Figure 10 shows the experimental setup used to test the stability. A square-wave signal, which is the input of the circuit under normal operating conditions, was connected to $v_{\text {in }}$. To display the potential instability of the circuit, the signal $v_{\mathrm{p}}$ was monitored by a digital oscilloscope (Agilent 54616C) via a $10 \times$ probe (with $10 \mathrm{M} \Omega \| 9 \mathrm{pF}$ input impedance). The circuit was considered unstable when a non-decreasing oscillation was superimposed on the exponential signal. The interconnection between the circuit and $C_{x}$ was implemented using coaxial cable for the signal path and one-wire cable for the return path. The one-wire cable was twisted along the coaxial cable in order to reduce the area of the current loop. The features of this interconnection cable were characterized using an impedance analyser (Agilent 4294A), and the results were $r_{\mathrm{p}}=1.0 \Omega \mathrm{m}^{-1}, c_{\mathrm{p}}=100 \mathrm{pF} \mathrm{m}^{-1}$ and $l_{\mathrm{p}}=1.0 \mu \mathrm{H} \mathrm{m}^{-1}$. The value of the resistor $R_{\mathrm{c}}$ was $100 \mathrm{k} \Omega$.

First, in order to validate the circuit model of the interconnection cable shown in figure $4(b)$, we tested the stability of, on the one hand, the circuit in figure 4(a) for $\ell=$ $5 \mathrm{~m}$ and, on the other hand, the circuit in figure $4(b)$ for $R_{\mathrm{p}}=$ $4.7 \Omega, C_{\mathrm{p}}=470 \mathrm{pF}$ and $L_{\mathrm{p}}=5 \mu \mathrm{H}$, which are approximately the parasitic components of a $5 \mathrm{~m}$ interconnection cable according to our model. Both circuits became unstable under the same test conditions. This means that the circuit model seems to be accurate enough to evaluate the stability of the circuit.

We tested the stability for several values of $C_{x}(10 \mathrm{pF}$, $47 \mathrm{pF}, 100 \mathrm{pF}$ and $470 \mathrm{pF}$, which were emulated by means of ceramic capacitors $), \ell(0 \mathrm{~m}, 0.5 \mathrm{~m}, 1 \mathrm{~m}$ and $5 \mathrm{~m})$ and $f_{\mathrm{b}}$ 
Table 2. Experimental results of the stability tests.

\begin{tabular}{llll}
\hline & \multicolumn{3}{c}{ Experimental instability cases } \\
\cline { 2 - 4 } OpAmp & $\ell=0.5 \mathrm{~m}$ & $\ell=1 \mathrm{~m}$ & $\ell=5 \mathrm{~m}$ \\
\hline OPA344 & Stable & Stable & Stable \\
OPA337 & Stable & Stable & Stable \\
OPA743 & Stable & Stable & Stable \\
TLC071 & Stable & $C_{x} \geqslant 470 \mathrm{pF}$ & $C_{x} \geqslant 100 \mathrm{pF}$ \\
AD8655 & $C_{x} \geqslant 470 \mathrm{pF}$ & $C_{x} \geqslant 100 \mathrm{pF}$ & $C_{x} \geqslant 47 \mathrm{pF}$ \\
OPA350 & $C_{x} \geqslant 47 \mathrm{pF}$ & $C_{x} \geqslant 47 \mathrm{pF}$ & $C_{x} \geqslant 47 \mathrm{pF}$ \\
\hline
\end{tabular}

(those listed in table 1). Table 2 summarizes the experimental results. For OPA344 and OPA337, the circuit was always stable. This is in agreement with figure 7 , because when $\ell \leqslant 5 \mathrm{~m}$ and $C_{x} \leqslant 470 \mathrm{pF}$, the critical frequency $f_{\text {stab }}$ is always greater than the bandwidth of these OpAmps. For TLC071 and OPA350, the circuit was unstable under certain conditions (see table 2), which is also predictable from figure 7 for $f_{\mathrm{b}}=$ $11 \mathrm{MHz}$ and $f_{\mathrm{b}}=64 \mathrm{MHz}$, respectively. However, for the other two OpAmps, some experimental results disagree with the theoretical predictions depicted in figure 7. For OPA743, the circuit should be unstable when $\ell=5 \mathrm{~m}$ and $C_{x}=470 \mathrm{pF}$, but experimentally it was stable. For AD8655, in addition to the cases listed in table 2, the circuit should also be unstable for (a) $\ell=0.5 \mathrm{~m}$ and $C_{x}=100 \mathrm{pF}$, and (b) $\ell=1 \mathrm{~m}$ and $C_{x}=$ $47 \mathrm{pF}$. These disagreements may be due to an OpAmp output resistance higher than $50 \Omega$, which is assumed in figure 7 (see section 3.1). If the actual value of $R_{\mathrm{o}}$ is higher than $50 \Omega$, then the circuit can be stable under conditions in which, according to figure 7 , it should be unstable.

Finally, we improved the stability of the circuit by connecting a $100 \Omega$ resistor in series with the OpAmp output. Using this resistor, we had fewer instability cases, as predicted in section 3.1. For example, for TLC071, the circuit was unstable only when $\ell \geqslant 5 \mathrm{~m}$ and $C_{x} \geqslant 470 \mathrm{pF}$. Regrettably, this additional resistor increases the effective output impedance of the OpAmp and, hence, can worsen the rejection of external interference, especially at high frequencies.

\subsection{Accuracy}

Figure 11 shows the experimental setup used to evaluate the error in the measurement of $C_{x}$ as caused by active shielding. A comparator-based relaxation oscillator was used as an interface circuit [11] and its output signal period was measured by a universal counter (Agilent 53131A). To avoid/reduce errors due to the non-idealities of the comparator, we used a highspeed comparator (TLV3501, 4.5 ns propagation delay) with a rail-to-rail output. We selected $R_{1}=R_{2}=R_{3}(=10 \mathrm{k} \Omega)$, so that $V_{\mathrm{TL}}=V_{\mathrm{DD}} / 3$ and $V_{\mathrm{TH}}=2 V_{\mathrm{DD}} / 3$, and hence the duty cycle of the output signal was $50 \%$. Other component values were $C_{x}=100 \mathrm{pF}, R_{\mathrm{c}}=100 \mathrm{k} \Omega$ and $V_{\mathrm{DD}}=5 \mathrm{~V}$.

First, a capacitor $C_{\mathrm{p}}$ was used instead of the interconnection cable, as shown in figure 11. The period of the oscillator output signal was measured for the six OpAmps listed in table 1 and for three values of $C_{\mathrm{p}}(0 \mathrm{pF}$, i.e. without any capacitor, $100 \mathrm{pF}$ and $200 \mathrm{pF}$ ). The case $C_{\mathrm{p}}=0 \mathrm{pF}$ was used as a reference to calculate the relative error of the period. Table 3 summarizes the resultant relative errors. On the one hand, the error for $C_{\mathrm{p}}=200 \mathrm{pF}$ was approximately twice that

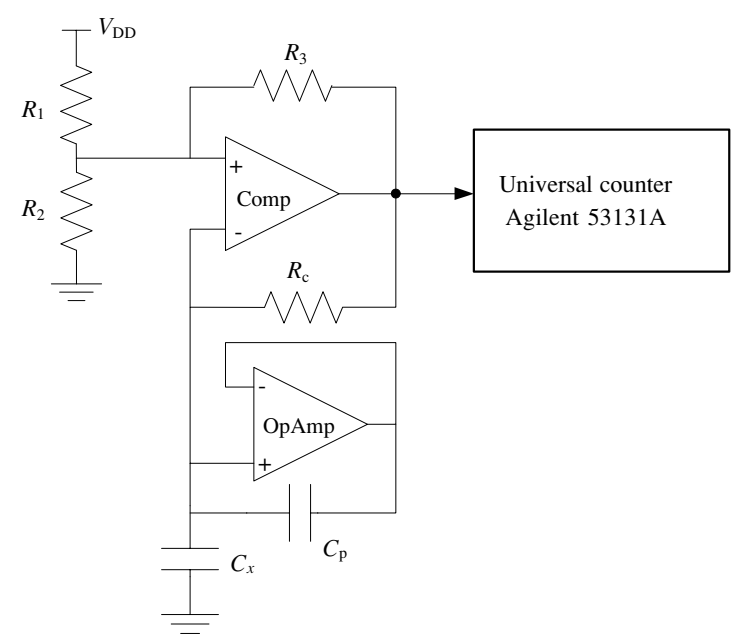

Figure 11. Experimental setup used to analyse the error in the measurement of $C_{x}$ as caused by active shielding.

Table 3. Experimental results of the accuracy tests.

\begin{tabular}{lll}
\hline & \multicolumn{2}{l}{ Experimental relative error $(\%)$} \\
\cline { 2 - 3 } OpAmp & $C_{\mathrm{p}}=100 \mathrm{pF}$ & $C_{\mathrm{p}}=200 \mathrm{pF}$ \\
\hline OPA344 & 1.53 & 3.21 \\
OPA337 & 0.58 & 1.09 \\
OPA743 & 0.39 & 0.62 \\
TLC071 & 0.24 & 0.44 \\
AD8655 & 0.14 & 0.24 \\
OPA350 & 0.11 & 0.19 \\
\hline
\end{tabular}

for $C_{\mathrm{p}}=100 \mathrm{pF}$, and, on the other hand, the error clearly decreased when the bandwidth of the amplifier increased. This performance agrees with the theoretical results shown in figure 9. Quantitatively, the experimental relative errors were slightly greater than the theoretical ones.

Finally, we measured the error in the period of the output signal when $C_{x}$ was connected to the interface circuit via a $1 \mathrm{~m}$ interconnection cable. For OPA344, OPA337, OPA743 and TLC071, the resulting errors were very similar to those shown in table 3 for the case $C_{\mathrm{p}}=100 \mathrm{pF}$, as expected since $c_{\mathrm{p}}=100 \mathrm{pF} \mathrm{m}^{-1}$. However, for AD8655 and OPA350, the error could not be measured because the circuit became unstable under such conditions, as indicated in section 4.1 as well. Therefore, the model developed in section 3.2 is helpful in predicting the error caused by active shielding whenever the circuit fulfils the stability condition described by equation (5).

\section{Conclusions}

The design of active shielding for grounded capacitive sensors requires special attention in order to prevent instability and inaccuracy. As the length of the shielded cable increases, so do the instability and the inaccuracy. However, an appropriate selection of the bandwidth of the amplifier that drives the shield of the coaxial cable can overcome these limitations. On the one hand, a wide bandwidth improves accuracy, but, on the other hand, a narrow bandwidth improves stability. Therefore, there is an optimal bandwidth range in terms of stability and accuracy. This is especially of interest 
for measurement systems intended for large/medium-value capacitive sensors. For low-value capacitive sensors (say, below $10 \mathrm{pF}$ ), no instability problems are expected since the critical frequency $f_{\text {stab }}$ is very high, and hence we can use a wide-bandwidth amplifier. Additional analysis is required for big-size capacitive sensors, such as liquid-level sensors for rivers or containers, since their parasitic components can also play an important role in the stability of the circuit.

\section{Acknowledgments}

This work was supported by the Dutch Technology Foundation (STW) under the project DET 6437 and by the Technical University of Catalonia through its foreign-stay programme.

\section{References}

[1] Baxter L K 1997 Capacitive Sensors. Design and Applications (New York: IEEE)

[2] Ross P J 1983 A water-level sensor using a capacitance to frequency converter J. Phys. E: Sci. Instrum. 16 827-8

[3] Bera S C, Ray J K and Chattopadhyay S 2006 A low-cost noncontact capacitance-type level transducer for a conducting liquid IEEE Trans. Instrum. Meas. 55 778-86

[4] Haase W C 2004 Digital measurement circuit and system using a grounded capacitive sensor US Patent Specification $6,700,392$
[5] Herzog M 1988 Circuit for measuring capacitance by charging and discharging capacitor under test and its shield US Patent Specification 4,743,837

[6] Huang S M, Stott A L, Green R G and Beck M S 1988 Electronic transducers for industrial measurement of low value capacitances J. Phys. E: Sci. Instrum. $21242-50$

[7] Marioli D, Sardini E and Taroni A 1993 High-accuracy measurement techniques for capacitance transducers Meas. Sci. Technol. 4 337-43

[8] Rich A 1983 Shielding and guarding Application Note AN-347 Analog Devices

[9] Morrison R 1995 Solving Interference Problems in Electronics (New York: Wiley)

[10] Pallas-Areny R and Webster J G 2001 Sensors and Signal Conditioning 2nd edn (New York: Wiley)

[11] Qi J, Meijer G C M, Li X and Guan C 2005 An integrated interface for grounded capacitive sensors Proc. 4th IEEE Sensors Conf. (Irvine, CA, USA)

[12] Franco S 2002 Design with Operational Amplifiers and Analog Integrated Circuits 3rd edn (New York: McGraw-Hill)

[13] Dorf R C and Bishop R H 2005 Modern Control Systems 10th edn (Upper Saddle River, NJ: Pearson-Prentice Hall)

[14] Mancini R 2001 Op Amps for Everyone. Design Reference Texas Instruments

[15] Goedbloed J J 1992 Electromagnetic Compatibility (New York: Prentice Hall)

[16] Meijer G C M, Van der Goes F M L, De Jong P C, Li X and Toth F N 1999 Integrated interfaces for low-cost multiple-sensor systems J. Intell. Mater. Syst. Struct. $10105-15$ 